\title{
Chronische Schmerzen nach Inguinalhernienchirurgie
}

Chronic Pain After Inguinal Hernia Surgery

Joël L. Lavanchy ${ }^{1}$, Konrad Streitberger ${ }^{2}$, Joel Zindel ${ }^{1}$, Guido Beldi ${ }^{1}$

Universitätsklinik für Viszerale Chirurgie und Medizin (1)

Universitätsklinik für Anästhesiologie und Schmerztherapie (2)

Inselspital, Universitätsspital Bern

3010 Bern, Schweiz

https://doi.org/10.1024/1661-8157/a002780 
Verwendete Abkürzungen:

NRS: Numeric rating scale

TAPP: transabdominale Hernienplastik

TEP: total extraperitoneale Hernienplastik

TENS: transcutaneous electric nerve stimulation

VAS: visual analogue scale

WHO: World Health Organisation

Zusammenfassung

Chronische Schmerzen nach Inguinalhernienoperationen sind häufig. Die aktuelle Evidenz zeigt, dass die endoskopische Versorgung von Hernien mit einem Netz chronische postoperative Schmerzen verringern. Dieser Review zeigt mögliche Ursachen und therapeutische Optionen bei Patienten mit chronischen Schmerzen auf.

\begin{abstract}
Chronic postoperative pain after inguinal hernia repair is common. Current evidence reveals a reduction of chronic postoperative pain after endoscopic mesh repair. This review describes diagnostic and therapeutic options for patients with chronic pain post hernia surgery.
\end{abstract}

Résumé

Des douleurs chroniques après l'opération d'une hernie inguinale se présentent souvent. L'évidence actuelle montre que l'implantation de filets lors de l'opération endoscopique, diminue l'apparition de douleurs chroniques postopératoires. Cette revue analyse les causes possibles et montre des options thérapeutiques pour les patients souffrants de douleurs chroniques suite à la chirurgie des hernies.

Schlüsselwörter

Chronische Schmerzen, Hernienchirurgie, Hernienversorgung, Inguinalhernie

Chronic pain, hernia surgery, hernia repair, inguinal hernia 
Douleur chronique, chirurgie des hernies, hernie inguinale 


\section{Hintergrund}

Inguinalhernienoperationen gehören mit einer Inzidenz von 100 bis 300 pro 100‘000 Einwohner und Jahr zu den häufigsten Operationen überhaupt (1). In der Schweiz werden jährlich mindestens 16‘000 Inguinalhernienoperationen durchgeführt (2). Chronische Schmerzen nach Inguinalhernienoperationen stellen heutzutage grössere Herausforderung dar als das Hernienrezidiv. Letzteres kann operativ mit Erfolg behoben werden, während chronische Schmerzen schwer zu therapieren sind. Hernienrezidive treten in weniger als 5\% der Patienten auf (1, 3-5). Dahingegen ist die Inzidenz von anhaltenden postoperativen Schmerzen nach Inguinalhernienoperationen zwischen verschiedenen prospektiven Studien mit 3-42\% tendenziell höher. Die Inzidenz ist variabel und hängt stark von der verwendeten Definition und Fragestellung (z.B. „Verspürten Sie in der letzten Woche ein Zwicken in der Leiste?“) ab (6-12). Abbildung 1 zeigt eine Auswahl relevanter Studien (6-33). Die Grössen der Kreise repräsentiert die Patientenanzahl der Studien, die Inzidenz von chronischen Schmerzen liegt insgesamt bei 21.8\% (gewichteter Mittelwert).

Insgesamt besteht eine Abnahmetendenz der chronischen Beschwerden mit jedem Jahr postoperativ (Abbildung 2) $(34,35)$. Aber Selbst acht Jahre nach einer Inguinalhernienoperation verspüren noch bis zu $23 \%$ der Patienten in der Woche vor der Untersuchung Schmerzen, davon ein Viertel der Patienten mittleren bis starken Ausmasses (34). Wichtig ist die Unterscheidung von leichten Schmerzen (gelegentliches Zwicken in der Leiste, Wetterfühligkeit), zu schweren Schmerzen, die zur Behinderung bei täglicher Aktivitäten bis hin zu Arbeitsunfähigkeit führen. Treten erstere bei jedem fünften Patienten auf, so sind zweitere mit einer Inzidenz von unter 6\% deutlich seltener (13). 
Die Wahrnehmung von postoperativen Schmerzen oder Beschwerden ist sehr individuell. Nozizeptive Schmerzen können von neuropathischen unterschieden werden. Erstere führen zu einer peripheren Sensibilisierung mit primärer und sekundärer Hyperalgesie und bei weiter gesteigerter Erregbarkeit der peripheren Nozizeptoren zu einer zentralen Sensibilisierung. Neuropathische Schmerzen als Folge von Nervenläsionen können sowohl eine verminderte Sensibilität (Hypästhesie) oder eine verstärkte Schmerzperzeption bei eigentlich nicht schmerzhaften Reizen (Allodynie) auslösen. Oft wird die Intensität von Beschwerden entweder mit der Verbalen Beschreibungsskala (verbal descriptor scale) in vier verschiedene Stufen (kein, milder, mittlerer oder starker Schmerz) unterteilt oder wird mit der Visuellen Analogskala (visual analogue scale: VAS) oder Numerischen Rating Skala (numeric rating scale: NRS) in elf möglichen Stufen von 0 bis 10 (alternativ 0 bis 100) evaluiert. Gemäss einer Publikation der International Association for the Study of Pain werden chronische postoperative Schmerzen definiert als Schmerzen, die sich nach einer Operation entwickeln und kontinuierlich für mindestens 2 Monate bestehen ohne dass eine andere Erklärung besteht $(12,36)$.

Nach Hernienoperationen wurden drei verschiedene Schmerzformen beschrieben: 1. Nozizeptiver Schmerz: Diese Schmerzform kann als Folge der inflammatorischen Reaktion auf den Operationsstimulus auftreten. Er wird zum Beispiel durch eine Reizung des Periosts verursacht (z.B. bei der Annaht des Netzes am Pecten ossis pubis bei der offenen Inguinalhernienoperation nach Lichtenstein). Der nozizeptive Schmerz ist die häufigste Schmerzform nach Hernienoperationen (37).

2. Neuropathischer Schmerz: Die direkte Schädigung eines Nervens löst diesen Schmerztyp unmittelbar aus (z.B. bei Tackerfixation des Netzes bei der total extraperitonealen Hernienplastik) (8) oder kann zur Bildung eines Neuroms führen, welches wiederum Ursache 
chronischer postoperativer Schmerzen sein kann (37).

3. Viszeraler Schmerz: Diese Schmerzqualität ist nach Hernienoperation selten und wird isoliert bei der Ejakulation beschrieben. Aetiologisch werden Vernarbungen des Ductus deferens oder Dyssynergien der ejakulatorischen Muskeln nach Verletzung somatischer oder sympathischer Nerven diskutiert (37).

Diese Schmerzformen können auch kombiniert auftreten (mixed pain) und zu zentralen Sensibilisierungsprozessen führen.

Im Folgenden werden Faktoren, welche Einfluss auf postoperative Schmerzen nach Hernienoperationen haben, identifiziert und diskutiert, wobei der Fokus dieser Übersicht bei den Inguinalhernien liegt.

Operationstechnik

Die Operationstechnik ist ein möglicher Einflussfaktor für postoperative Schmerzen. Hierbei stehen drei Fragen im Vordergrund:

1. Hat ein Netz (Kunststoffimplantat) einen Einfluss auf postoperative Schmerzen? Traditionellerweise wurden Inguinalhernien ohne die Einlage eines Netzes (Operation nach Bassini, Operation nach Shouldice) behoben. Die Versorgung mit einem spannungsfrei einlegten Netz (Operation nach Lichtenstein) wurde 1987 eingeführt (38). Multiple randomisierte Studien und ein Cochrane Review zeigen weniger chronischen Schmerzen nach Hernienoperationen mit Implantation eines Netzes als bei Techniken ohne Netzimplantation (39-41). Als mögliche Ursache finden ist die Raffung der Faszien bei netzfreien Operationen (Operation nach Bassini oder Shouldice) welche für die Stabilität der Bauchwand nötig ist. Bei Operationen mit Netz 
(Operation nach Lichtenstein) entfällt die Raffung, da ein Netz spannungsfrei eingelegt wird.

2. Lassen sich chronische postoperative Schmerzen durch eine minimal invasive Operation reduzieren?

Randomisierte Studien und systematische Reviews zeigen, dass endoskopische Techniken wie die laparoskopische transabdominale Hernienplastik (TAPP) oder die total extraperitoneale Hernienplastik (TEP) im Vergleich zur offenen Inguinalhernienoperation weniger chronische Schmerzen erzeugen (10, 41-44). Die TAPP und die TEP sind bezüglich den postoperativen Resultaten vergleichbar (45).

3. Welches ist das optimale chirurgische Management von Nerven während der Operation?

Identifikation und Schonung von Nerven vermeidet chronische Schmerzen bei offener und endoskopischer Operationen. Die primäre Resektion von Nerven scheint kurzfristig mit weniger Schmerzen einher zu gehen, der langfristige Nutzen ist nicht gesichert (46).

\section{Netzmaterial}

Aufgrund der deutlich geringeren Rezidivraten und geringeren Schmerzen werden heutzutage in der Hernienchirurgie standardmässig Netze implantiert (39). Ein zusätzlicher Einfluss von Netzmaterial auf die Entwicklung von Schmerzen ist möglich, da je nach Struktur und Zusammensetzung eine unterschiedliche ausgeprägte Entzündungsreaktion und Narbenbildung entsteht. Den Standard bilden Polypropylen-Netze, welche durch eine Fremdkörperreaktion zu einer Vernarbung und somit zur Stabilisierung der Region um die Bruchlücke führen. Netze mit einem reduzierten Polypropylen-Anteil (sogenannte lightweight Netze) wurden deshalb zur Reduktion der lokalen Entzündungsreaktion und des 
postoperativen Schmerzes untersucht. In zwei Metaanalysen von neun randomisierten Studien ( $n=2310$ ) der offenen Inguinalhernienversorgung wurde ein geringeres Auftreten von chronischen postoperativen Schmerzen beim Verwenden von «lightweight mesh» im Vergleich zu «heavyweight mesh» nachgewiesen (48) (49). Beim endoskopischen Vorgehen sind zwei Metaanalysen weniger konzis, die Verwendung eines «leightweight mesh» kann jedoch ebenfalls empfohlen werden $(50,51)$

\section{Netzbefestigung}

Generell wird davon ausgegangen, dass die Penetration der Bauchwand durch die Netzfixation postoperative Schmerzen verursacht. So wurde die Netzfixation mit Tackern in der endoskopischen Hernienchirurgie als mögliche Ursache von Nervenirritationen und Osteitiden diskutiert (9). Bei kleinen, insbesondere bei lateralen, Hernien soll das Netz ohne Befestigung eingebracht werden. Die aktuelle Datenlage lässt jedoch auch hier keine klare Empfehlungen für eine bestimmte Fixationsart zu $(52,53)$. Ein möglicher Trend zu weniger Schmerzen bei Reduktion des Fixationstraumas ist wahrscheinlich. Die Interpretation der Daten ist jedoch erschwert aufgrund der Tatsache, dass die Netzfixation auch abhängig von der Grösse der Bruchlücke ist. Bei zunehmender Grösse der Bruchlücke ist eine straffere und rigidere Fixation des Netzes indiziert.

\section{Prädiktive Faktoren}

Haupt-Risikofaktoren für die Entwicklung chronischer Schmerzen nach Hernienoperationen sind starke postoperative Schmerzen (54), ein hohes präoperatives Schmerzniveau und postoperative Komplikationenn (Tabelle 1) (13). 
Tabelle 1: Allgemeine Risikofaktoren für postoperative chronische Schmerzen

Diagnostik von chronische postoperativen Schmerzen

Postoperative Schmerzen im Bereich des Eingriffes können vielfältige Ursachen haben. Eine vorübergehende Hypästhesie rund um die Operationsnarbe tritt häufig auf und gehört zum normalen Verlauf der Wundheilung. Eine Hyperalgesie oder Allodynie hingegen, vor allem wenn sie im postoperativen Verlauf persistiert oder gar zunimmt, sollte zu weiterführenden Abklärungen führen. Ein Hernienrezidiv als mögliche Ursache der Beschwerden ist selten, weil es häufig nicht schmerzhaft ist. Entsprechend sind die Patienten präoperativ aufzuklären. Bei postoperativen Schmerzen nach Inguinalhernienoperationen sollte bei entsprechend klinischem Korrelat auch konventionell-radiologisch eine Hüftpathologie (Coxarthrose, Schenkelhalsfraktur, Femurkopfnekrose, etc.) ausgeschlossen werden. Die Magnetresonanztomographie kann Schmerzursachen, welche nicht operationsspezifisch sind (Arthritiden, Bursitiden, urogentiale Ursachen), identifizieren, liefert jedoch selten Aufschluss über operationsspezifische Gründe für Leistenschmerzen (55).

Um das weitere Chronifizierungsrisiko einschätzen zu können, sollten möglichst früh in die Diagnose die psychosoziale Situation des Patienten (v.a. Ängstlichkeit, psychische Anfälligkeit, Arbeitsplatzsituation, familiäres Umfeld) und ggf. vor der Operation bestehende Schmerzprobleme mit einbezogen werden. Am besten erfolgt dies sofern möglich bereits präoperativ. Bei bereits präoperativ bestehenden chronischen Schmerzen in der Region der Hernie sollte die Operationsindikation mit Bedacht und Zurückhaltung gestellt werden. Eine Hernie ist selten die Ursache von Schmerzen in der Leiste. Mit der Hernienoperation kann zwar die Hernie behoben werden, das chronische Schmerzproblem persistiert jedoch häufig nach der Operation. 
Therapie

Zur Therapie postoperativer Schmerzen empfiehlt sich ein stufenweises Vorgehen. Parallel soll nach anatomischen Ursachen (insbesondere ein Hernienrezidiv) gesucht, und eine Therapie begonnen werden. Eine ausreichende Analgesie nach WHO-Stufenschema bildet hierfür von Anfang an die Basis. Eine gute schmerzhemmende Grundlage bieten hierbei Metamizol und Nichtsteroidale Antirheumatika. Insbesondere in der früh-postoperativen Phase sollten frühzeitig, aber auch möglichst kurzfristig, Opioidanalgetika eingesetzt werden.

Hernien-Rezidive sollten bei Schmerzen in jedem Fall reoperiert werden. Ein anteriorer Zugangsweg ist in jedem Fall empfohlen (auch wenn bereits der Primäreingriff anterior erfolgte). Zudem soll eine Tripple-Neurektomie proximal der Ursachenstelle erfolgen (56).

Bei fehlendem Erfolg der üblichen Analgetika und Verdacht auf neuropathische Schmerzkomponenten kann der Einsatz von Ketamin, Gabapentinoiden (Pregabalin oder Gabapentin) oder schmerzmodulierenden trizyklischen Antidepressiva (z.B. Trimipramin oder Amitryptilin) in Betracht gezogen werden.

Physiotherapie: Eine häufige Ursache postoperativer Schmerzen ist eine Dekonditionierung der Bauchdecke. Dem liegt eine prolongierte Schonung und Inaktivierung der Rumpfmuskulatur zugrunde. Eine Schonung von über 14 Tagen nach Leistenhernienoperationen ist in der Regel nicht angezeigt. Aus diesem Grunde soll in einem ersten Schritt ein gezielter Aufbau der Rumpfmuskulatur durch Physiotherapie erfolgen. Die Begleitung dieser Massnahme durch myofasziale Behandlungstechniken zum Beispiel mit Manueller Medizin kann erfolgreich sein. 
Infiltrationen: Eine häufige Ursache postoperativer Schmerzen sind anhaltende lokalen Nervenirritationen und die Ausbildung eines Neurinoms. Diagnostisch ist eine anatomisch klar abgrenzbare Symptomatik welche dem Verlauf eines Nerven (N. iliohypogastricus, N. ilioinguinalis oder N. genitofemoralis) entspricht. Ein elektrisierender Schmerz durch Beklopfen (Tinel Zeichen) ist in dieser Situation möglich. Lokale Infiltrationen mit langwirksamen Lokalanästhetika (Bupivacain, Ropivacain) bieten sich sowohl diagnostisch wie auch therapeutisch an. Infiltrationen können in einem ersten Schritt ohne sonographische Kontrolle auf die Ebene der Faszie und gegebenenfalls intramuskulär lateral der muskulären Nervendurchtrittsstelle erfolgen. Werden durch eine Injektion die Schmerzen temporär behoben, so ist an neurogenen Schmerz zu denken. Dies kann dann gegebenenfalls mittels ultraschallgezielter Nerveninfiltration z.B. des N. ilioinguinalis, N. ilioingunialis oder N. genitofemoralis abgeklärt werden. Oft kann eine Injektion auch zu langanhaltendem Erfolg führen weil dadurch sowohl eine Bahnung der nozizeptiven Nervenfasern wie auch muskuläre Irritationen gelöst werden können. Einer Chronifizierung des Schmerzes kann so Einhalt geboten werden. Auch eine Behandlung mit TENS (Transcutaneous electric nerve stimulation) oder Akupunktur kann bei persistierenden neurogenen Schmerzen zu einer erfolgreichen positiven Schmerzmodulation führen.

Neuroablative Verfahren: Bei persistierenden Schmerzen und Identifizierung eines speziellen Nerven nach kurzfristig erfolgreichen diagnostischen Infiltrationen können ablative Verfahren wie Kryotherapie und Radiofrequenzablation in Betracht gezogen werden. Ultima ratio ist die chirurgische Neurektomie sensibler Nervenäste oder die operative Revision der Hernie mit Explantation des Netzes. Die Indikation zu Neuroablativen Verfahren sollte allerdings bei ausgeprägten psychosozialen Risikofaktoren für eine Schmerzchronifizierung und bei einem Verdacht auf zentrale Sensibilisierung zurückhaltend gestellt werden. In diesem Fall sollte unbedingt ein Schmerzspezialist mit involviert und die Möglichkeiten eines multimodalen Schmerztherapieprogramms evaluiert werden. 
Schmerzedukation: Eine frühzeitige Aufklärung über den normalen Schmerzverlauf und den Umgang mit anhaltenden Schmerzen kann die weitere Therapie wesentlich erleichtern. Bei bereits bestehenden chronischen Schmerzen können zudem psychologische Therapieansätze, Entspannungsverfahren und Schmerzmanagementkurse empfohlen werden.

Prophylaxe: Die beste Prophylaxe ist neben einem schonenden Operationsverfahren eine gute und individuell angepasste postoperative Schmerztherapie. Wenn bereits präoperativ Risiken einer möglichen Chronifizierung (insbesondere vorbestehende chronischen Schmerzen) evaluiert werden, ist dies umso wichtiger. Dabei kann perioperativ auch der Einsatz von Ketamin oder Gabapentinoiden in Betracht gezogen werden.

Fazit

Chronische Schmerzen nach Operationen bei Inguinalhernien sind häufig und werden durch die Verwendung endoskopischer Operationstechniken reduziert. Chronifizierende therapiebedürftige Schmerzen sind jedoch selten und sollten interdisziplinär in Zusammenarbeit zwischen Hausarzt, Chirurg und Schmerztherapeut behandelt werden. Erste Behandlungsschritte erfolgen mit Physiotherapie, manueller Medizin gefolgt von Infiltrationen mit Lokalanästhesie, TENS, am besten auch im Rahmen einer Multimodalen Schmerztherapie. Nur bei Versagen aller konservativer und interventioneller Behandlungsversuche erfolgt unter Berücksichtigung der psychosozialen Situation die Prüfung neuroablativer Verfahren. 
Key messages

- Chronische Schmerzen nach Hernienoperationen sind häufig.

- Risikofaktoren sind vorbestehende präoperative Schmerzen, starke postoperative Schmerzen, genetische und psychosoziale Faktoren

- Es treten drei Schmerzformen auf: nozizeptiver, neuropathischer und viszeraler Schmerz.

- Bei chronischen Schmerzen spielen zentrale Sensibilisierungsprozesse eine wesentliche Rolle

- Sowohl die Einlage eines Netzes, als auch endoskopische Operationstechniken verringern chronische postoperative Schmerzen.

- Die Therapie chronischer postoperativer Schmerzen nach Hernienoperationen ist multimodal und interdisziplinär. 


\section{Lernfragen}

1. In wieviel Prozent der Patienten treten nach Inguinalhernienoperationen chronische Schmerzen auf?
A) $1 \%-5 \%$
B) $7 \%-11 \%$
C) $11 \%-29 \%$
D) $30 \%-34 \%$

2. Welches ist/sind die häufigste/n Schmerzform/en nach Hernienoperationen?
A) viszerale und nozizeptive Scherzen
B) nozizeptiver Schmerz
C) viszeraler Schmerz
D) neuropathischer Schmerz

3. Welche Faktoren verringern die Wahrscheinlichkeit chronischer postoperativer Schmerzen nach Hernienoperationen?
A) präoperative Schmerzen
B) lightweight Netze
C) Tackerlose Fixation des Netzes
D) endoskopische Verfahren

4. Bei Versagen von Analgesie und Physiotherapie welches ist der nächste Schritt der therapeutischen Optionen bei postoperativen Schmerzen nach Hernienoperationen?
A) Netzexplantation
B) lokale Infiltrationen
C) selektive Neurolyse
D) Durchführung eines MRI

Richtige Antworten: 1. C, 2. B, 3. D, 4. B

Korrespondenzadresse:

Prof. Dr. med. Guido Beldi

Universitätsklinik für Viszerale Chirurgie und Medizin

Inselspital, Universitätsspital Bern

3010 Bern, Schweiz

guido.beldi@insel.ch 
Abbildung 1: Anzahl Patienten mit chronischen Schmerzen. Die Grösse der Kreise entspricht der Anzahl eingeschlossenen Patienten.

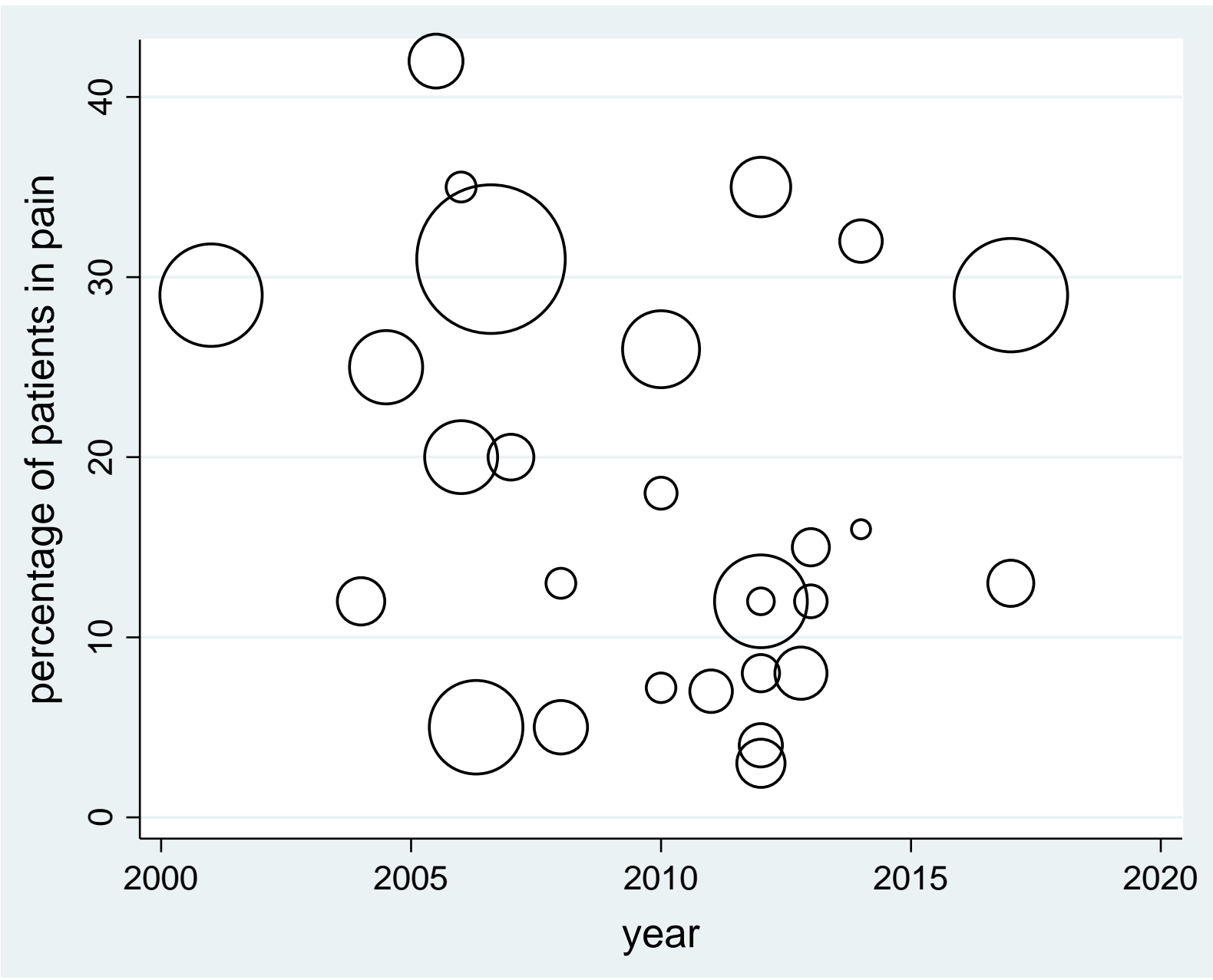


Abbildung 2: Verlauf der chronischen Schmerzen postoperativ. Die Summe aller Studie sowie Studien die mehrere Endpunkte vergleichen zeigen eine stete, wenn auch langsame, Abnahme der chronischen Schmerzen mit der Zeit.

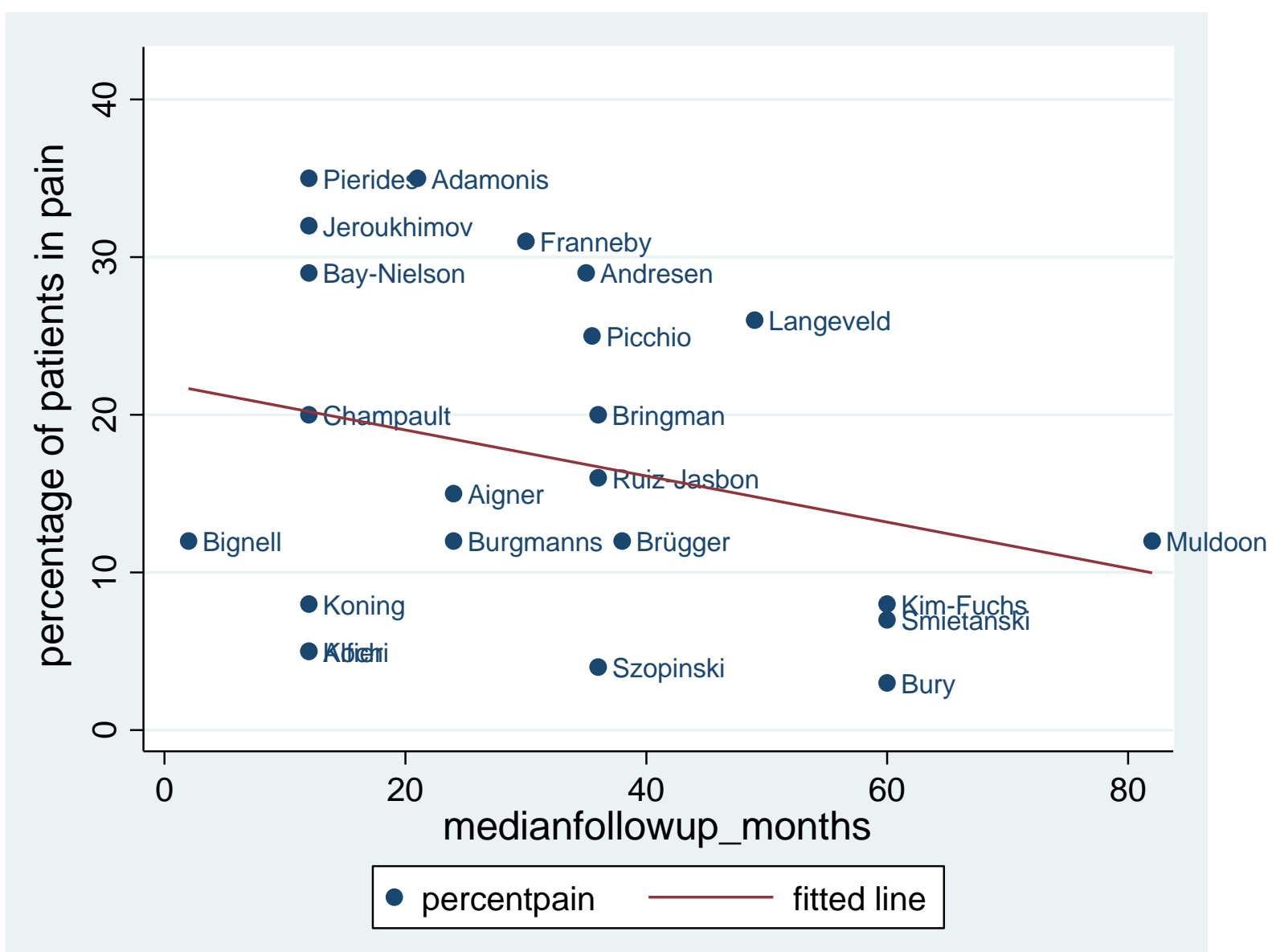




\section{Referenzen:}

1. Kingsnorth A, LeBlanc K. Hernias: inguinal and incisional. Lancet. 2003;362(9395):1561-1571.

2. Schneider T, Langenegger M, Hofstetter K, et al. Qualitätsindikatoren der Schweizer Akutspitäler. In: Schweizerische Eidgenossenschaft EDdl, Bundesamt für Gesundheit, ed; 2016.

3. O'Reilly EA, Burke JP, O'Connell PR. A meta-analysis of surgical morbidity and recurrence after laparoscopic and open repair of primary unilateral inguinal hernia. Ann Surg. 2012;255(5):846-853.

4. Willaert W, De Bacquer D, Rogiers X, et al. Open Preperitoneal Techniques versus Lichtenstein Repair for elective Inguinal Hernias. Cochrane Database Syst Rev. 2012(7):CD008034.

5. de Goede B, Klitsie PJ, van Kempen BJ, et al. Meta-analysis of glue versus sutured mesh fixation for Lichtenstein inguinal hernia repair. Br J Surg. 2013;100(6):735-742.

6. Eklund A, Montgomery A, Bergkvist L, et al. Chronic pain 5 years after randomized comparison of laparoscopic and Lichtenstein inguinal hernia repair. Br J Surg. 2010;97(4):600608.

7. Langeveld HR, van't Riet $M$, Weidema WF, et al. Total extraperitoneal inguinal hernia repair compared with Lichtenstein (the LEVEL-Trial): a randomized controlled trial. Ann Surg. 2010;251(5):819-824.

8. Callesen T, Bech K, Kehlet H. Prospective study of chronic pain after groin hernia repair. Br J Surg. 1999;86(12):1528-1531.

9. Poobalan AS, Bruce J, Smith WC, et al. A review of chronic pain after inguinal herniorrhaphy. Clin J Pain. 2003;19(1):48-54.

10. Nienhuijs S, Staal E, Strobbe L, et al. Chronic pain after mesh repair of inguinal hernia: a systematic review. Am J Surg. 2007;194(3):394-400.

11. Bay-Nielsen $M$, Perkins $F M$, Kehlet $H$, et al. Pain and functional impairment 1 year after inguinal herniorrhaphy: a nationwide questionnaire study. Ann Surg. 2001;233(1):1-7.

12. Schug SA, Pogatzki-Zahn EM. Chronic Pain after Surgery or Injury. Pain Clinical Updates. 2011;19(1):1-5.

13. Franneby $U$, Sandblom $G$, Nordin $P$, et al. Risk factors for long-term pain after hernia surgery. Ann Surg. 2006;244(2):212-219.

14. Bury K, Smietanski M. Five-year results of a randomized clinical trial comparing a polypropylene mesh with a poliglecaprone and polypropylene composite mesh for inguinal hernioplasty. Hernia. 2012;16(5):549-553.

15. Szopinski J, Dabrowiecki S, Pierscinski S, et al. Desarda versus Lichtenstein technique for primary inguinal hernia treatment: 3-year results of a randomized clinical trial. World J Surg. 2012;36(5):984-992.

16. Alfieri S, Rotondi F, Di Giorgio A, et al. Influence of preservation versus division of ilioinguinal, iliohypogastric, and genital nerves during open mesh herniorrhaphy: prospective multicentric study of chronic pain. Ann Surg. 2006;243(4):553-558.

17. Koch A, Bringman S, Myrelid P, et al. Randomized clinical trial of groin hernia repair with titanium-coated lightweight mesh compared with standard polypropylene mesh. Br J Surg. 2008;95(10):1226-1231.

18. Smietanski M, Bury K, Smietanska IA, et al. Five-year results of a randomised controlled multicentre study comparing heavy-weight knitted versus low-weight, non-woven polypropylene implants in Lichtenstein hernioplasty. Hernia. 2011;15(5):495-501.

19. Kim-Fuchs $C$, Angst $E$, Vorburger $S$, et al. Prospective randomized trial comparing sutured with sutureless mesh fixation for Lichtenstein hernia repair: long-term results. Hernia.

2012;16(1):21-27. 
20. Koning GG, Keus F, Koeslag L, et al. Randomized clinical trial of chronic pain after the transinguinal preperitoneal technique compared with Lichtenstein's method for inguinal hernia repair. Br J Surg. 2012;99(10):1365-1373.

21. Muldoon RL, Marchant K, Johnson DD, et al. Lichtenstein vs anterior preperitoneal prosthetic mesh placement in open inguinal hernia repair: a prospective, randomized trial. Hernia. 2004;8(2):98-103.

22. Brugger $\mathrm{L}$, Bloesch $\mathrm{M}$, Ipaktchi $\mathrm{R}$, et al. Objective hypoesthesia and pain after transabdominal preperitoneal hernioplasty: a prospective, randomized study comparing tissue adhesive versus spiral tacks. Surg Endosc. 2012;26(4):1079-1085.

23. Bignell $M$, Partridge $G$, Mahon $D$, et al. Prospective randomized trial of laparoscopic (transabdominal preperitoneal-TAPP) versus open (mesh) repair for bilateral and recurrent inguinal hernia: incidence of chronic groin pain and impact on quality of life: results of 10 year follow-up. Hernia. 2012;16(6):635-640.

24. Burgmans JP, Voorbrood CE, Simmermacher RK, et al. Long-term Results of a Randomized Double-blinded Prospective Trial of a Lightweight (Ultrapro) Versus a Heavyweight Mesh (Prolene) in Laparoscopic Total Extraperitoneal Inguinal Hernia Repair (TULP-trial). Ann Surg. 2016;263(5):862-866.

25. Aigner F, Augustin F, Kaufmann C, et al. Prospective, randomized-controlled trial comparing postoperative pain after plug and patch open repair with totally extraperitoneal inguinal hernia repair. Hernia. 2014;18(2):237-242.

26. Ruiz-Jasbon F, Norrby J, Ivarsson ML, et al. Inguinal hernia repair using a synthetic long-term resorbable mesh: results from a 3-year prospective safety and performance study. Hernia. 2014;18(5):723-730.

27. Bringman S, Wollert S, Osterberg J, et al. Three-year results of a randomized clinical trial of lightweight or standard polypropylene mesh in Lichtenstein repair of primary inguinal hernia. Br J Surg. 2006;93(9):1056-1059.

28. Picchio $M$, Palimento $D$, Attanasio $U$, et al. Randomized controlled trial of preservation or elective division of ilioinguinal nerve on open inguinal hernia repair with polypropylene mesh. Arch Surg. 2004;139(7):755-758; discussion 759.

29. Andresen $\mathrm{K}$, Fenger $\mathrm{AQ}$, Burcharth J, et al. Mesh fixation methods and chronic pain after transabdominal preperitoneal (TAPP) inguinal hernia surgery: a comparison between fibrin sealant and tacks. Surg Endosc. 2017.

30. Jeroukhimov I, Wiser I, Karasic E, et al. Reduced postoperative chronic pain after tension-free inguinal hernia repair using absorbable sutures: a single-blind randomized clinical trial. J Am Coll Surg. 2014;218(1):102-107.

31. Adamonis $W$, Witkowski $P$, Smietanski $M$, et al. Is there a need for a mesh plug in inguinal hernia repair? Randomized, prospective study of the use of Hertra 1 mesh compared to PerFix Plug. Hernia. 2006;10(3):223-228.

32. Pierides $G$, Scheinin $T$, Remes $V$, et al. Randomized comparison of self-fixating and sutured mesh in open inguinal hernia repair. Br J Surg. 2012;99(5):630-636.

33. O'Dwyer PJ, Kingsnorth AN, Molloy RG, et al. Randomized clinical trial assessing impact of a lightweight or heavyweight mesh on chronic pain after inguinal hernia repair. Br J Surg. 2005;92(2):166-170.

34. Nienhuijs SW, Rosman C. Long-term outcome after randomizing prolene hernia system, mesh plug repair and Lichtenstein for inguinal hernia repair. Hernia. 2015;19(1):77-81.

35. Dahlstrand U, Sandblom G, Nordin P, et al. Chronic pain after femoral hernia repair: a crosssectional study. Ann Surg. 2011;254(6):1017-1021. 
36. Classification of chronic pain. Descriptions of chronic pain syndromes and definitions of pain terms. Prepared by the International Association for the Study of Pain, Subcommittee on Taxonomy. Pain Suppl. 1986;3:S1-226.

37. Cunningham J, Temple WJ, Mitchell P, et al. Cooperative hernia study. Pain in the postrepair patient. Ann Surg. 1996;224(5):598-602.

38. Lichtenstein IL. Herniorrhaphy. A personal experience with 6,321 cases. Am J Surg. 1987;153(6):553-559.

39. Scott NW, McCormack K, Graham P, et al. Open mesh versus non-mesh for repair of femoral and inguinal hernia. Cochrane Database Syst Rev. 2002(4):CD002197.

40. Grant AM, Collaboration EUHT. Open mesh versus non-mesh repair of groin hernia: metaanalysis of randomised trials based on individual patient data [corrected]. Hernia. 2002;6(3):130-136.

41. Collaboration EUHT. Repair of groin hernia with synthetic mesh: meta-analysis of randomized controlled trials. Ann Surg. 2002;235(3):322-332.

42. McCormack K, Scott NW, Go PM, et al. Laparoscopic techniques versus open techniques for inguinal hernia repair. Cochrane Database Syst Rev. 2003(1):CD001785.

43. Neumayer L, Giobbie-Hurder A, Jonasson O, et al. Open mesh versus laparoscopic mesh repair of inguinal hernia. N Engl J Med. 2004;350(18):1819-1827.

44. Bringman S, Ramel S, Heikkinen TJ, et al. Tension-free inguinal hernia repair: TEP versus mesh-plug versus Lichtenstein: a prospective randomized controlled trial. Ann Surg. 2003;237(1):142-147.

45. Bracale U, Melillo P, Pignata G, et al. Which is the best laparoscopic approach for inguinal hernia repair: TEP or TAPP? A systematic review of the literature with a network metaanalysis. Surg Endosc. 2012;26(12):3355-3366.

46. Barazanchi AW, Fagan PV, Smith BB, et al. Routine Neurectomy of Inguinal Nerves During Open Onlay Mesh Hernia Repair: A Meta-analysis of Randomized Trials. Ann Surg. 2016;264(1):64-72.

47. Bringman $\mathrm{S}$, Wollert $\mathrm{S}$, Osterberg J, et al. One year results of a randomised controlled multicentre study comparing Prolene and Vypro II-mesh in Lichtenstein hernioplasty. Hernia. 2005;9(3):223-227.

48. Sajid MS, Leaver C, Baig MK, et al. Systematic review and meta-analysis of the use of lightweight versus heavyweight mesh in open inguinal hernia repair. Br J Surg. 2012;99(1):2937.

49. Uzzaman MM, Ratnasingham K, Ashraf N. Meta-analysis of randomized controlled trials comparing lightweight and heavyweight mesh for Lichtenstein inguinal hernia repair. Hernia. 2012;16(5):505-518.

50. Currie A, Andrew H, Tonsi A, et al. Lightweight versus heavyweight mesh in laparoscopic inguinal hernia repair: a meta-analysis. Surg Endosc. 2012;26(8):2126-2133.

51. Sajid MS, Kalra L, Parampalli U, et al. A systematic review and meta-analysis evaluating the effectiveness of lightweight mesh against heavyweight mesh in influencing the incidence of chronic groin pain following laparoscopic inguinal hernia repair. Am J Surg. 2013;205(6):726736.

52. Hoyuela $\mathrm{C}$, Juvany $\mathrm{M}$, Carvajal $\mathrm{F}$, et al. Randomized clinical trial of mesh fixation with glue or sutures for Lichtenstein hernia repair. Br J Surg. 2017.

53. Sun $\mathrm{P}$, Cheng $\mathrm{X}$, Deng $\mathrm{S}$, et al. Mesh fixation with glue versus suture for chronic pain and recurrence in Lichtenstein inguinal hernioplasty. Cochrane Database Syst Rev. 2017;2:CD010814.

54. Olsson A, Sandblom G, Franneby U, et al. Impact of postoperative complications on the risk for chronic groin pain after open inguinal hernia repair. Surgery. 2016. 
55. Burgmans JP, Voorbrood CE, Van Dalen T, et al. Chronic pain after TEP inguinal hernia repair, does MRI reveal a cause? Hernia. 2016;20(1):55-62.

56. Lange JFM, Kaufmann R, Wijsmuller AR, et al. An international consensus algorithm for management of chronic postoperative inguinal pain. Hernia. 2015;19(1):33-43.

57. Schnabel A, Pogatzki-Zahn E. [Predictors of chronic pain following surgery. What do we know?]. Schmerz. 2010;24(5):517-531; quiz 532-513.

58. Macintyre PE, Schug SA, Scott DA, et al. APM:SE Working Group of the Australian and

New Zealand College of Anaesthetists and Faculty of Pain Medicine (2010), Acute Pain Management: Scientific Evidence (3rd edition), ANZCA \& FPM, Melbourne.

Tabelle 1

Allgemeine Risikofaktoren für postoperative chronische Schmerzen (nach $(57,58))$ :

- Präoperative Schmerzen über 1 Monat

- Revisionseingriffe

- Psychische Anfälligkeit (Katastrophisieren)

- Präoperative Ängstlichkeit

- Frauen und jüngere Erwachsene

- IV-Rentenverfahren

- Genetische Prädisposition

- Verminderte körpereigene Schmerzhemmung (diffuse noxious inhibitory control)

- Erhöhtes Eingriffsrisiko für Nervenschaden

- Akute starke ungenügend behandelte Schmerzen

- Jüngeres Alter (10)

- Ausschliesslich Lokalanästhesie (10) 